\title{
Continuous Thread Metal Container Closure
}

National Cancer Institute

\section{Source}

National Cancer Institute. Continuous Thread Metal Container Closure. NCI Thesaurus.

Code C96115.

Metal closure turned onto a corresponding thread on the top or mouth of a container, whether it be glass, plastic or metal. 